\title{
Telepresence-teleguidance to facilitate training and quality assurance in ERCP: a health economic modeling approach
}

\section{(ㄷ)(1) $\ominus$}

\author{
Authors \\ Institutions \\ 1 Innovation Centre, Division of Innovation and \\ Development, Karolinska University Hospital, \\ Stockholm, Sweden \\ 2 Department of Surgery, Centre for Digestive Diseases, \\ Karolinska University Hospital, Stockholm, Sweden \\ 3 CLINTEC, Karolinska Institutet, Stockholm Sweden \\ 4 Department of Surgery, Odense University Hospital, J.B. \\ Winsloews Vej 4, 5000 Odense, Denmark
}

Johanna Brinne Roos ${ }^{1}$, Per Bergenzaun ${ }^{2}$, Kristina Groth ${ }^{1}$, Lars Lundell ${ }^{2,3,4}$, Urban Arnelo ${ }^{2,3}$

submitted 13.5.2019

accepted after revision $\quad 16.10 .2019$

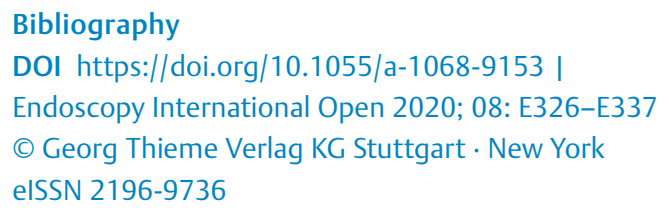

Corresponding author

Lars Lundell MD, PhD, Department of Surgery, CLINTEC, Karolinska Institute, Stockholm, Sweden

Fax: +46-8-58586721

lars.lundell@ki.se

Appendix

Online content viewable at:

https://doi.org/10.1055/a-1068-9153

\section{ABSTRACT}

Background and study aims The aims of this study was to document the clinical and training relevance of endoscopic retrograde cholangiopancreaticography (ERCP) teleguidance (as a clinical model for applied telemedicine) with health economic modeling methodologies.

Methods Probabilities and consequences of complications after ERCP performed by either a novice-trainee or supported through teleguidance (TM) by an expert formed the basis of the health economic model.

Results The main clinical and economic outcomes originated from the base case scenario representing a low-volume center. In the cohort the patient age was 62 years, $58 \%$ were females, the expert was doing $\geq 250$ ERCPs per year and 50 for the novice-trainee. The expert knowledge transferred was set to $50 \%$ and the average complexity grade to 1.98. Given a willingness to pay threshold of 56,180 USD/ quality-adjusted life years (QALY), the probability of costeffectiveness of TM assistance was $98.9 \%$. The probability of a QALY gain for patients having an ERCP, to which was added TM, was $91.6 \%$. Adding TM saved on an average 111.2 USD ( $95 \%$ CI 959 to 1021 SEK) per patient, and remained cost-effective basically insensitive to the level of willingness to pay.

Conclusion Teleguidance during an ERCP procedure has the potential to be the prefered option in many low- to medium-volume hospitals. The main mechanisms behind these effects are positive impact on several adverse patient outcomes, QALY increase, and decreased costs. TM should be considered for integration into future teaching curriculums in advanced upper gastrointestinal endoscopy.

\section{Introduction}

Technology-enabled health care at a distance has profound scientific and commercial potential and accordingly has been met with growing interest. The term telemedicine generally means provision and delivery of health care using telecommunication tools. Its recent application in the medical field means that medical information is exchanged from one site to another to provide health care provider with the purpose of improving patient care, including consultative, diagnostic and Treatment services [1]. Similarly, technological progress has allowed medicine and/or teaching to be practiced, without direct physical physician - patient or physician - student interactions [2].

In the field of advanced gastrointestinal endoscopy, the endoscopic view is displayed on a high-definition screen, whereupon, for example, a trainee can be safely guided by a senior colleague, an application of telemedicine usually referred to as teleguidance. Moreover, digitalization of images has greatly facilitated communication between and development of professional networks in medicine in general and in endos- 
copy in particular. Video image transmissions during endoscopic retrograde cholangiopancreaticography (ERCP) have been utilized for postgraduate teaching and found useful for live demonstrations of procedures performed by experts [3$6]$. In one study it was demonstrated that use of telemedicine even offered the option of enhanced quality of ERCP in a lowvolume hospital, provided that teleguidance was provided by a tertiary referral center [7], resulting in an increased cannulation rate (an alleged proxy for quality of care), at the low volume hospital, from $85 \%$ to $97 \%$. The current status of the available telemedicine solution has reached the level where connecting and support are parts of daily routine practice. Accordingly the telemedicine solution and its implementation now seem to be ready for a more widespread use. Indeed, the technique has great potential to be practiced in other areas of medicine, for example, in endoluminal vascular interventions and in the emergency room to enhance coordination and support in management of trauma. However, one pivotal aspect to be considered, but often neglected when introducing new technologies, is considering the health economic aspects. The objective of the current study, therefore, was to document the relevance of ERCP teleguidance (as a clinical model) using health economic modeling methodologies.

\section{Methods}

A probabilistic health economic model was constructed to investigate for what type of hospital and under which conditions teleguidance during ERCP could be an attractive option. The primary outcome measures were cost savings and quality adjusted life year (QALY) increase. QALY represents an instrument generally applied, by which one can weigh different medical efforts against each other. The idea is based on the fact that one should take into account not only how many years extra that different medical efforts can give, but also the quality of those years. A complete healthy person is considered to have a QALY value of 1 and death as a consequence of the intervention has a value of 0 . One year in full health corresponds to $1 \mathrm{QALY}$. The model was constructed to take into account all discernible clinical management options in management of these patients, which are displayed in detail in $>$ Fig. $\mathbf{1} \mathbf{a}$ and $\mathbf{b}$.

Highlighted in these figures are also the time points, during clinical management of the patients, where teleguidance might diminish risk of adverse outcomes and alternatively, increase likelihood of success. All these considerations were entirely based on expert opinions. Again based on similar expert opinion, to which was added actual data captured in the relevant literature [8-34], teleguidance was investigated concerning its potential effect on cannulation failure/success, complication rates, mortality following a putative surgical intervention, duration (minutes) of the individual ERCP procedure, as well as the position of the investigator on the respective learning curve.

The overall effect of teleguidance was considered to be dependent on the amount of expert knowledge transferred via the actual teleguidance procedure. The outcomes for the novice-trainee endoscopist will ultimately approach those of the expert, the more expert knowledge is transferred. This can be mathematically described where the equation derived is depicted in Formula $\mathbf{1}$ in the appendix.

In the health economic model, predictive analytics can be applied to determine probability of complications after ERCP. To perform the analysis, we used outcomes presented in a systematic review of prospective studies completed by Andriulli et al [8], including 16,855 patients. Accordingly, we created four variable logistic regression models, given in Formula 2 (see appendix), for calculating the probability of having post-ERCP complications such as pancreatitis, bleeding, perforation, infection or even a lethal outcome.

We used the collected complication data [8] to construct the predictive models to be integrated in the final health economic model. The Number of patients per study included in that review ranged from 210 to 2,769 and was used for internal weighing in the models. The Annual Number of procedures performed by the endoscopist at each center varied from 26 to 212. Using these data, we estimated a rank that represented the mean difficulty-rank of the procedures performed at each center. To estimate this rank, we created a three-variable logistic regression model based on the total Number of complications in each individual study cohort. Based on the difference between the actual outcome in a respective study and what the three variable logistic models could predict, the final rank was defined. If the difference for one study fell below the $33 \mathrm{rd}$ percentile of the distribution of difference in all studies, the rank was given the value of 1 . When this difference was between the 33rd and the 66th percentiles, the rank was 2 and otherwise it was given the value of 3 . Adding the rank as the 4th variable in the logistic regression model, for the total Number of complications, we obtained the four-variable logistic predictive model the outcomes of which are presented in $>$ Table 1 , together with the logistic regression models constructed for post-ERCP pancreatitis, bleeding, perforation, infection, and death.

The centers in this table correspond to the parameter values $\left(\overline{\chi_{1}}, \overline{\chi_{2}}, \overline{\chi_{3}}, \overline{\chi_{4}}\right)$ from Formula 2 as detailed above. Inserting $\left(\chi_{1}, \chi_{2}, \chi_{3}, \chi_{4}\right)=\left(\overline{\chi_{1}}, \overline{\chi_{2}}, \overline{\chi_{3}}, \overline{\chi_{4}}\right)$ into this equation, where each outcome variable equals to the mean probabilities captured from the systematic literature review [8]. The centers $\left(\overline{\chi_{1}}, \overline{\chi_{2}}, \overline{\chi_{3}}, \overline{\chi_{4}}\right)$ were calculated by numerically solving the equation $\left.p\left(\chi_{1}, \chi_{2}, \chi_{3}, \chi_{4}\right)-p_{\text {average }}=0\right)$

This health economic model considered only the direct medical costs, from the perspective of the health care provider, at a low- to medium-volume hospital. Moreover, the time horizon for the model was restricted to 1 year because the majority of outcomes relevant to an ERCP procedure present themselves within such a time frame [8-34]. In the same model, shortterm outcomes and associated costs were handled within the decision-tree structure, as described above, and they were then used to populate the first-month cycle in a Markov model as displayed in the Fig. $\mathbf{s} \mathbf{1}$ enclosed in the appendix.

Recurrent and/or late complications after hospital discharge, until the end of the first post-procedural year, are excluded in this Markov model. To simplify the health economic modeling further, utilities were only assigned to the different 


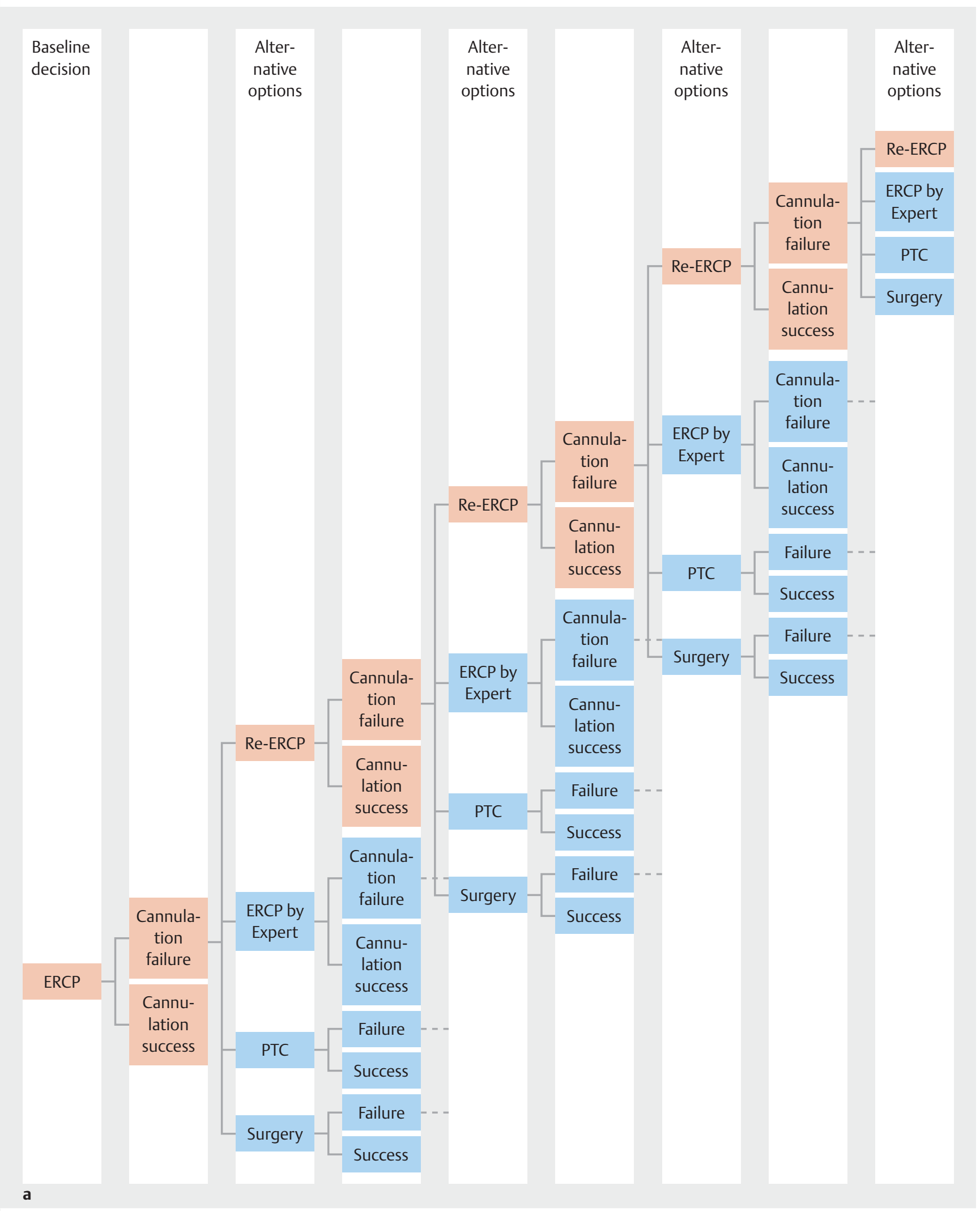

- Fig. 1a Decision tree for the indication, occurrence and types of reinterventions in case of cannulation failure at the ERCP investigation. In case of failure all alternative options can be chosen as the next step in the model . ERCP stands for Endoscopic Retrograde Cholangio Pacreaticography and PTC for Percutaneous Transhepatic Cholangiography. 


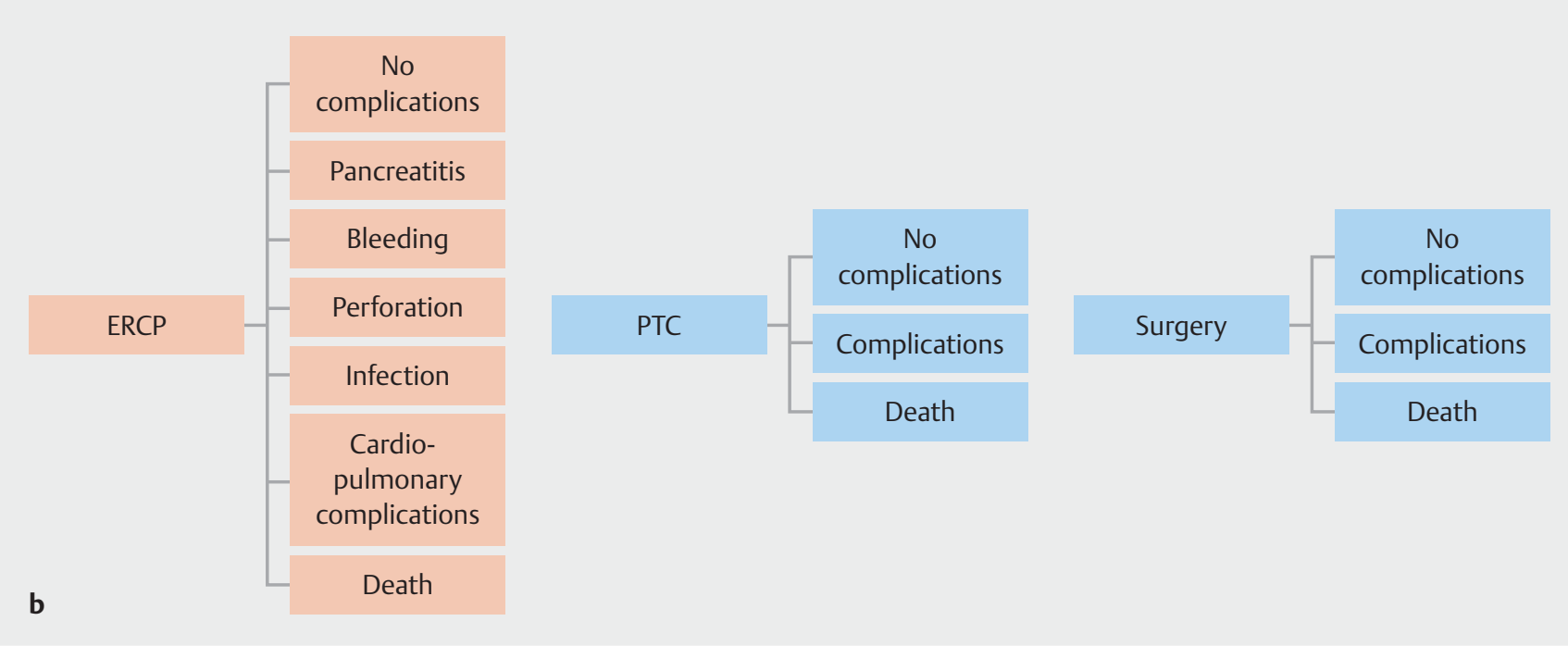

- Fig. 1b Decision tree for the indication, occurrence and types of reinterventions in case of cannulation failure at the ERCP investigation. In case of failure all alternative options can be chosen as the next step in the model . In b is depicted the clinical outcomes as defined and implemented in the model. ERCP stands for Endoscopic Retrograde Cholangio Pacreaticography and PTC for Percutaneous Transhepatic Cholangiography.

Table 1a Key probabilities for the base case patient cohort.

\begin{tabular}{|l|l|l|l|}
\hline & Expert & Novice & TM \\
\hline Cannulation rate ERCP & 0.980629 & 0.854112 & 0.917370 \\
\hline Pancreatitis & 0.026543 & 0.037425 & 0.031984 \\
\hline Bleeding & 0.009080 & 0.015267 & 0.012173 \\
\hline Perforation & 0.007275 & 0.005294 & 0.006284 \\
\hline Infection & 0.005954 & 0.019145 & 0.012549 \\
\hline Cardiopulmonary complications & 0.013335 & 0.013335 & 0.013335 \\
\hline ERCP Death & 0.002551 & 0.003466 & 0.003008 \\
\hline No Complications & 0.935258 & 0.906068 & 0.920663 \\
\hline TM, teleguidance-assisted procedure; ERCP, endoscopic retrograde cholangiopancreaography. & & \\
\hline
\end{tabular}

Table 1b Corresponding probabilities for endoscopists performing $50 \mathrm{ERCP} / \mathrm{year}$ with mean difficulty 1 (scale 1-4). where the TM probabilities are calculated for $25 \% .50 \%$ and $75 \%$ expert knowledge transferred. respectively.

\begin{tabular}{|l|l|l|l|l|l|}
\hline & Expert & Novice & TM 25\% & TM 50\% & TM 75\% \\
\hline Cannulation rate ERCP & 0.985797331 & 0.889220319 & 0.913364572 & 0.937508825 & 0.961653078 \\
\hline Pancreatitis & 0.020878489 & 0.029507862 & 0.027350519 & 0.025193176 & 0.023035832 \\
\hline Bleeding & 0.007361713 & 0.01239188 & 0.011134338 & 0.009876796 & 0.008619254 \\
\hline Perforation & 0.005388399 & 0.00391852 & 0.00428599 & 0.00465346 & 0.005020929 \\
\hline Infection & 0.003598623 & 0.01163047 & 0.009622509 & 0.007614547 & 0.005606585 \\
\hline $\begin{array}{l}\text { Cardiopulmonary } \\
\text { complications }\end{array}$ & 0.013335389 & 0.013335389 & 0.013335389 & 0.013335389 & 0.013335389 \\
\hline ERCP Death & 0.001924138 & 0.002614733 & 0.002442084 & 0.002269436 & 0.002096787 \\
\hline No complications & 0.947513249 & 0.926601146 & 0.931829171 & 0.937057197 & 0.942285223 \\
\hline TM, teleguidance-assisted procedure; ERCP, endoscopic retrograde cholangiopancreaography. & & & \\
\hline
\end{tabular}


- Table 1c As above with mean difficulty 2. in d) the mean difficulty 3. in e) with a mean difficulty 4. in $\mathbf{f}$ ) where the endoscopists performing 100 ERCP per year with mean difficulty 1 . in $\mathbf{g}$ ) where the endoscopists performing 100 ERCP per year with mean difficulty 2 . in $\mathbf{h}$ ) where the endoscopists performing 100 ERCP per year with mean difficulty 3 and finaly in i) where endoscopists performed 100 ERCP per year with mean difficulty 4 .

\begin{tabular}{|c|c|c|c|c|c|}
\hline & Expert & Novice & TM $25 \%$ & TM $50 \%$ & TM $75 \%$ \\
\hline Cannulation rate ERCP & 0.980506687 & 0.853307166 & 0.885107047 & 0.916906927 & 0.948706807 \\
\hline Pancreatitis & 0.026673569 & 0.037606136 & 0.034872994 & 0.032139852 & 0.029406711 \\
\hline Bleeding & 0.009119354 & 0.015331946 & 0.013778798 & 0.01222565 & 0.010672502 \\
\hline Perforation & 0.007320173 & 0.005326155 & 0.005824659 & 0.006323164 & 0.006821668 \\
\hline Infection & 0.006016426 & 0.01933988 & 0.016009017 & 0.012678153 & 0.00934729 \\
\hline $\begin{array}{l}\text { Cardiopulmonary } \\
\text { complications }\end{array}$ & 0.013335389 & 0.013335389 & 0.013335389 & 0.013335389 & 0.013335389 \\
\hline ERCP Death & 0.002565921 & 0.003486054 & 0.00325602 & 0.003025987 & 0.002795954 \\
\hline No complications & 0.934969169 & 0.905574441 & 0.912923123 & 0.920271805 & 0.927620487 \\
\hline
\end{tabular}

TM, teleguidance-assisted procedure; ERCP, endoscopic retrograde cholangiopancreaography.

$>$ Table 1d For details see legend to $>$ Table $1 \mathrm{c}$.

\begin{tabular}{|c|c|c|c|c|c|}
\hline & Expert & Novice & TM $25 \%$ & TM $50 \%$ & TM $75 \%$ \\
\hline Cannulation rate ERCP & 0.973298604 & 0.808261913 & 0.849521086 & 0.890780259 & 0.932039431 \\
\hline Pancreatitis & 0.034021254 & 0.04781743 & 0.044368386 & 0.040919342 & 0.037470298 \\
\hline Bleeding & 0.011291866 & 0.018956175 & 0.017040098 & 0.015124021 & 0.013207944 \\
\hline Perforation & 0.009937578 & 0.007235776 & 0.007911226 & 0.008586677 & 0.009262128 \\
\hline Infection & 0.010042304 & 0.03199415 & 0.026506188 & 0.021018227 & 0.015530265 \\
\hline $\begin{array}{l}\text { Cardiopulmonary } \\
\text { complications }\end{array}$ & 0.013335389 & 0.013335389 & 0.013335389 & 0.013335389 & 0.013335389 \\
\hline ERCP Death & 0.003421031 & 0.004646376 & 0.00434004 & 0.004033703 & 0.003727367 \\
\hline No Complications & 0.917950577 & 0.876014705 & 0.886498673 & 0.896982641 & 0.907466609 \\
\hline
\end{tabular}

TM, teleguidance-assisted procedure; ERCP, endoscopic retrograde cholangiopancreaography.

$\longrightarrow$ Table 1e For details see legend to $\$ Table $1 \mathrm{c}$.

\begin{tabular}{|l|l|l|l|l|l|}
\hline & Expert & Novice & TM 25\% & TM 50\% & TM 75\% \\
\hline Cannulation rate ERCP & 0.963524327 & 0.753382208 & 0.805917737 & 0.858453267 & 0.910988797 \\
\hline Pancreatitis & 0.043302935 & 0.060626755 & 0.0562958 & 0.051964845 & 0.04763389 \\
\hline Bleeding & 0.013974639 & 0.023416742 & 0.021056216 & 0.01869569 & 0.016335165 \\
\hline Perforation & 0.013478159 & 0.009823303 & 0.010737017 & 0.011650731 & 0.012564445 \\
\hline Infection & 0.016716782 & 0.052485093 & 0.043543015 & 0.034600937 & 0.02565886 \\
\hline $\begin{array}{l}\text { Cardiopulmonary } \\
\text { Complications }\end{array}$ & 0.013335389 & 0.013335389 & 0.013335389 & 0.013335389 & 0.013335389 \\
\hline ERCP Death & & & & 0.005375159 & 0.004967485 \\
\hline No complications & 0.00455981 & 0.006190509 & 0.005782834 & 0.864377248 & 0.879504767 \\
\hline
\end{tabular}

TM, teleguidance-assisted procedure; ERCP, endoscopic retrograde cholangiopancreaography. 
$\triangleright$ Table $1 \mathrm{f}$ For details see legend to $\triangleright$ Table $1 \mathrm{c}$.

\begin{tabular}{|l|l|l|l|l|l|}
\hline & Expert & Novice & TM 25\% & TM 50\% & TM 75\% \\
\hline Cannulation rate ERCP & 0.985797331 & 0.936137509 & 0.948552465 & 0.96096742 & 0.973382376 \\
\hline Pancreatitis & 0.020878489 & 0.027071107 & 0.025522952 & 0.023974798 & 0.022426644 \\
\hline Bleeding & 0.007361713 & 0.010882019 & 0.010001942 & 0.009121866 & 0.008241789 \\
\hline Perforation & 0.005388399 & 0.004243509 & 0.004529732 & 0.004815954 & 0.005102177 \\
\hline Infection & 0.003598623 & 0.008682539 & 0.00741156 & 0.006140581 & 0.004869602 \\
\hline $\begin{array}{l}\text { Cardiopulmonary } \\
\text { complications }\end{array}$ & 0.013335389 & 0.013335389 & 0.013335389 & 0.013335389 & 0.013335389 \\
\hline ERCP Death & 0.001924138 & 0.002421801 & 0.002297385 & 0.00217297 & 0.002048554 \\
\hline No Complications & 0.947513249 & 0.933363637 & 0.93690104 & 0.940438443 & 0.943975846 \\
\hline TM, teleguidance-assisted procedure; ERCP, endoscopic retrograde cholangiopancreaography. & & & \\
\hline
\end{tabular}

- Table 1g For details see legend to $>$ Table $1 \mathrm{c}$.

\begin{tabular}{|l|l|l|l|l|l|}
\hline & Expert & Novice & TM 25\% & TM 50\% & TM 75 \\
\hline Cannulation rate ERCP & 0.980506687 & 0.913962577 & 0.930598605 & 0.947234632 & 0.96387066 \\
\hline Pancreatitis & 0.026673569 & 0.034524416 & 0.032561704 & 0.030598993 & 0.028636281 \\
\hline Bleeding & 0.009119354 & 0.013468744 & 0.012381397 & 0.011294049 & 0.010206702 \\
\hline Perforation & 0.007320173 & 0.005767212 & 0.006155452 & 0.006543692 & 0.006931932 \\
\hline Infection & 0.006016426 & 0.014466474 & 0.012353962 & 0.01024145 & 0.008128938 \\
\hline $\begin{array}{l}\text { Cardiopulmonary } \\
\text { complications }\end{array}$ & 0.013335389 & 0.013335389 & 0.013335389 & 0.013335389 & 0.013335389 \\
\hline ERCP death & 0.002565921 & 0.003229038 & 0.003063259 & 0.002897479 & 0.0027317 \\
\hline No Complications & 0.934969169 & 0.915208727 & 0.920148837 & 0.925088948 & 0.930029058 \\
\hline TM, teleguidance-assisted procedure; ERCP, endoscopic retrograde cholangiopancreaography. & & & \\
\hline
\end{tabular}

$\checkmark$ Table $1 \mathrm{~h}$ For details see legend to $>$ Table $1 \mathrm{c}$.

\begin{tabular}{|l|l|l|l|l|l|}
\hline & Expert & Novice & TM 25\% & TM 50\% & TM 75\% \\
\hline Cannulation rate ERCP & 0.973298604 & 0.885033465 & 0.90709975 & 0.929166035 & 0.95123232 \\
\hline Pancreatitis & 0.034021254 & 0.043937126 & 0.041458158 & 0.03897919 & 0.036500222 \\
\hline Bleeding & 0.011291866 & 0.016659994 & 0.015317962 & 0.01397593 & 0.012633898 \\
\hline Perforation & 0.009937578 & 0.007833723 & 0.008359687 & 0.008885651 & 0.009411615 \\
\hline Infection & 0.010042304 & 0.024010099 & 0.02051815 & 0.017026201 & 0.013534253 \\
\hline $\begin{array}{l}\text { Cardiopulmonary } \\
\text { Complications }\end{array}$ & 0.013335389 & 0.013335389 & 0.013335389 & 0.013335389 & 0.013335389 \\
\hline ERCP death & 0.003421031 & 0.004304183 & 0.004083395 & 0.003862607 & 0.003641819 \\
\hline No complications & 0.917950577 & 0.889919487 & 0.89692726 & 0.903935032 & 0.910942805 \\
\hline TM, teleguidance-assisted procedure; ERCP, endoscopic retrograde cholangiopancreaography. & & & \\
\hline
\end{tabular}




\begin{tabular}{|c|c|c|c|c|c|}
\hline & Expert & Novice & TM $25 \%$ & TM $50 \%$ & TM $75 \%$ \\
\hline Cannulation rate ERCP & 0.963524327 & 0.847995019 & 0.876877346 & 0.905759673 & 0.934642 \\
\hline Pancreatitis & 0.043302935 & 0.055767875 & 0.05265164 & 0.049535405 & 0.04641917 \\
\hline Bleeding & 0.013974639 & 0.020591589 & 0.018937351 & 0.017283114 & 0.015628876 \\
\hline Perforation & 0.013478159 & 0.010632787 & 0.01134413 & 0.012055473 & 0.012766816 \\
\hline Infection & 0.016716782 & 0.039596753 & 0.03387676 & 0.028156767 & 0.022436775 \\
\hline $\begin{array}{l}\text { Cardiopulmonary } \\
\text { complications }\end{array}$ & 0.013335389 & 0.013335389 & 0.013335389 & 0.013335389 & 0.013335389 \\
\hline ERCP death & 0.00455981 & 0.00573525 & 0.00544139 & 0.00514753 & 0.00485367 \\
\hline No complications & 0.894632287 & 0.854340358 & 0.86441334 & 0.874486322 & 0.884559305 \\
\hline
\end{tabular}

some patients might have experienced more severe complications than others and even more so during a longer-time span. Health state values, or "utilities," are clinically useful because they measure outcomes in a single, common metric form, allowing meaningful comparison across diseases and interventions. In addition, no further costs were assigned to cycles 2 to 12 of the Markov model and mortality from the second postoperative month onward was estimated based on the average age and gender-related mortality figures taken from the matched general population of Sweden [35].

A hypothesis was formulated that teleguidance would shift the sloop of the learning curve to the left, which is reflected by higher cannulation rates and successful completion of more complex ERCP procedures at the low- to medium-volume hospital. For comparison, in case of unavailable teleguidance, more costly therapeutic strategies, such as reintervention in the form of open surgery and/or referral to a high volume center, have to be pursued.

For the part of the health economic model, which takes into account the teleguidance effect on the learning curve for the trainee endoscopist, we used actual data from a low-volume hospital [7]. Absent teleguidance support, the cannulation rate for the novice-trainee during the subsequent year would reach an intermediate value of $92 \%$. Similarly when offering teleguidance support to the learning curve, the novice-trainee with an initial cannulation rate of $85 \%$ will attain a cannulation rate close to the level of an expert after 5 years. Without such assistance it was assumed that this level of expertise was reached first after 10 years. The area between these two learning curves, as displayed in $>$ Fig. 2 a, relates to the cost savings associated with teleguidance support, essentially being due to a decrease in number of reinterventions and changed strategy following a cannulation failure.

In the model, adherence to a similar learning curve will impact costs. To construct the traditional and teleguidance (TM) learning curves, we defined the novice-trainee as an endoscopist performing 50 ERCPs per year with an average difficulty score of 2 (on a scale from 1-4). Procedure length for such a novice-trainee was estimated to be approximately 1.5 times long- er, according to opinions of experts at Karolinska University Hospital. The procedure length factor was assumed to change linearly with the number of ERCPs carried out per year as was the shape of the traditional and TM learning curves, respectively. The latter was allegedly dependent on the initial cannulation rate of the novice-trainee again co-varying with the number of ERCPs carried out per year.

The learning curve of the novice trainee in $>$ Fig. 2a will have one slope if the trainee has teleguidance support until he or she has reached the same cannulation rate as an expert at t_TM. If the teleguidance support ends before the trainee has reached the level of an expert, the area between the curves will be smaller. How the area between the curves depends on the expert support time t, can be seen in - Fig. 2b. t_conventional in this figure is the time it will take to reach the cannulation rate of an expert without teleguidance support. Area A will increase with support time $\mathrm{t}$ from 0 to $\mathrm{t}_{-} \mathrm{TM}$. At $\mathrm{t}=\mathrm{t}$-TM the area $\mathrm{A}$ has reached its maximum and will plateau. Since the depreciation time of the equipment traditionally is 3 to 5 years the support time $t$ is set to 3 years in the model. For further details and equations used to calculate corresponding cost savings see Formula 3 in the appendix.

A large number of different variables including probabilities, costs variables and Utility estimates were used in the model, the details of which are given in Tables1-s10 in the appendix. The various scenario parameters of the model were used to investigate the circumstances under which teleguidance would become a preferable option as a function of the different conditions and center sizes (the different scenario parameter for the base case are given in Table $\mathbf{s} 3$ in the appendix). When the cost data were retrieved from non-Swedish studies, the costs were converted to 2016 SEK values using the established conversion and inflation factors (for details see appendix). Because we only considered a 1-year time horizon in the model, discounting of costs and outcomes was omitted.

The average cannulation rate for an expert at Karolinska University hospital is traditionally close to $95 \%$, but the complexity of the procedures performed at this hospital is higher than in low- to moderate-volume hospitals. In our previous study [7], 

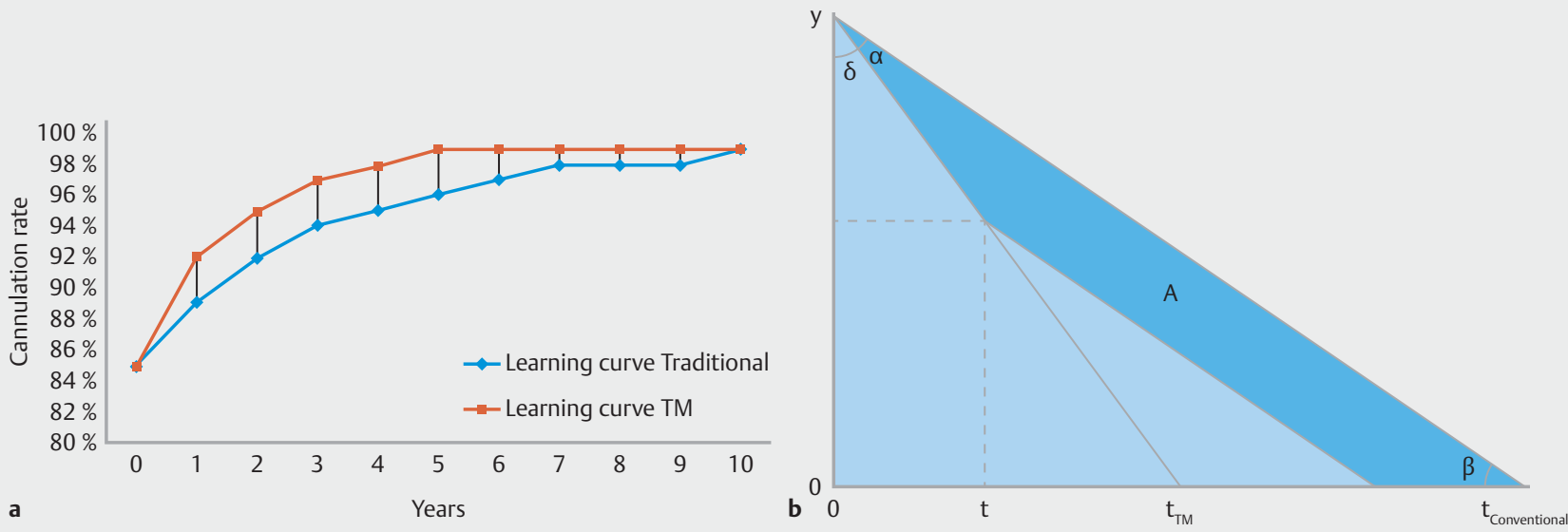

- Fig. 2 The slopes of the learning curve for successful ERCP by the presence or absence of teleguidance (TM). In $\mathbf{b}$ is illustrated the various assumptions and their consequences on the learning effects on direct medical costs confined to ERCP. ERCP stands for Endoscopic Retrograde Cholangio Pacreaticography.

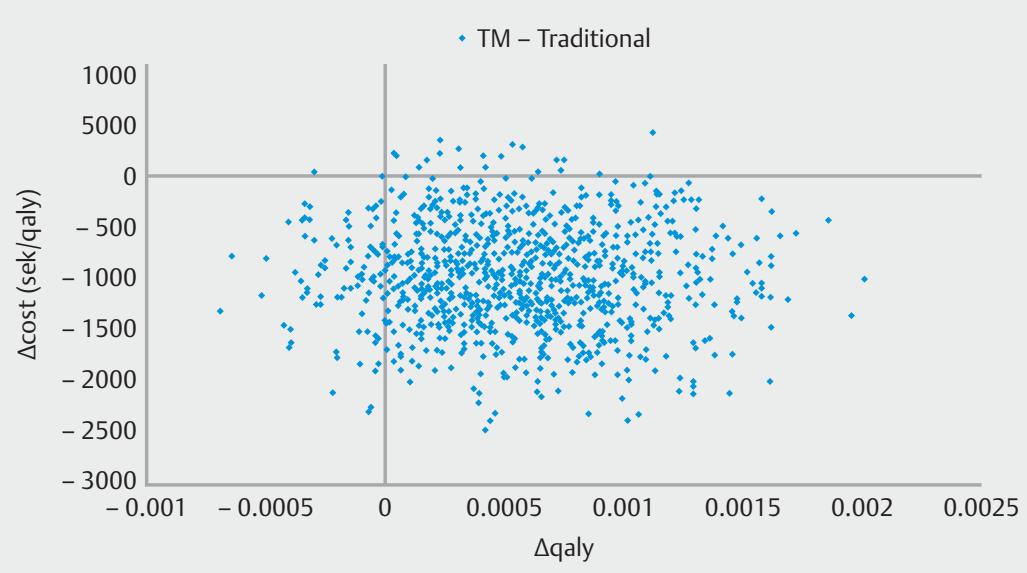

- Fig. 3 Cost Effectiveness Plane for Teleguided (TM) ERCP vs traditional approach to ERCP. ERCP stands for Endoscopic Retrograde Cholangio Pacreaticography.

the endoscopist rated the value of assistance through teleguidance, on a scale from 0 to 2, giving an average value of 0.92 for 26 consecutive procedures. These two outcomes suggested that the expert knowledge transferred will be in the interval between $46 \%$ and $100 \%$. If equal weight was given both to how the novice-trainee endoscopist experienced the guidance and the increased rate of successful cannulations, the corresponding value would converge to $73 \%$. However, in the base case scenario, the value for the expert knowledge transferred during the teleguidance, was conservatively chosen to be as low as $50 \%$. In the base case scenario, among the 50 ERCP procedures at the peripheral (low-volume) hospital, 16 were scored to be of complexity grade 1, 22 of grade 2, nine of grade 3 and three of complexity grade 4 , resulting in an average complexity score of 1.98. The relationship between clinical complexity and rank, used in the prediction models, is given by the equation Rank= Difficulty $3 / 4$. The reintervention strategy chosen for the base case scenario, in case of failed initial ERCP, was to repeat the ERCP and then, as a second step, to do percutaneous-transhepatic-cholangiography (PTC) and thereafter send the patient to an expert. This represents the most common management strategy for a low- to medium-volume hospital, according to the opinions of the experts. The model was, however, built to handle all possible options as displayed in $>$ Fig. 1. Multivariate sensitivity analysis was performed using Monte Carlo simulation and was presented for the base case scenario and for six hypothetical centers covering nine different conditions. One thousand simulations were performed in each case to model the most common and possible outcomes. 


\section{Results}

The main clinical and economic outcomes originate from the base case scenario hospital, a hospital which represented a low-volume center having one endoscopist performing 50 ERCP procedures per year. At this unit the strategy is, in case of cannulation failure, to proceed with a redo ERCP and then to a (PTC) and if still unsuccessful send the patient to an expert. In this base case, the average complexity grade was set to 1.98 and the amount of expert knowledge transferred during teleguidance to $50 \%$. The ensuing cost-effectiveness outcomes are visualized in the cost-effectiveness plane in > Fig. $\mathbf{3}$ (in table format see supplement).

For the base case scenario the cohort the patient age was 62 years, the percentage females attained $58 \%$, the number of ERCPs/year for the expert 250 and those for the novice-trainee 50 . Hereby the percentage of expert knowledge transferred amounted to only $50 \%$, the procedure difficulty was 1,98 and in case of ERCP failure the strategy was followed to do PTC and then send the patient to an expert. Given these inputs the prediction models (and using Equation 1 in the appendix) gave the following values for the expert: Cannulation rate $=98.1 \%$, postERCP pancreatitis rate $=2.65 \%$, bleeding rate $=0.91 \%$, perforation rate $=0.73 \%$, infection rate $=0.60 \%$, cardiopulmonary complications rate $=1.33 \%$ and lethal outcome in $0.25 \%$ of the cases. For the novice-trainee the same prediction model gave the following values: cannulation rate $=85.4 \%$, post-ERCP pancreatitis rate $=3.74 \%$, bleeding rate $=1.53 \%$, perforation rate $=$ $0.53 \%$, infection rate $=1.91 \%$, cardiopulmonary complications rate $=1.33 \%$ and death rate $=0.35 \%$. When $50 \%$ of the expert knowledge was transferred, through teleguidance to the novice trainee, the following outcomes emerged: cannulation rate $=$ $91.7 \%$, post-ERCP pancreatitis rate $=3.20 \%$, bleeding rate $=$ $1.21 \%$, perforation rate $=0.63 \%$, infection rate $=1.26 \%$, cardiopulmonary complications rate $=1.33 \%$ and a death rate of $0.30 \%$. The deterministic health economics, following the traditional novice trainee program, resulted in a procedure cost of 28,890 SEK ( 3246 USD) with a QALY of 0,9203 , whereas following teleguidance the corresponding values were; 27960 SEK (3137 USD) and 0.9209, respectively. Further details examplifying the interrelationsship between e.g. cannulation rate, the impact of TM and cost savings are given in Table $\mathbf{1 1}$ (appendix)

Given a willingness to pay threshold of 500,000 SEK $(56,180$ USD)/QALY, the probability of cost-effectiveness for the base case scenario was found to be $98.9 \%$. In fact our calculations suggested that TM was cost effective irrespective of the level of the willingness to pay. These results showed that the probability of a QALY gain, for patients that undergo an ERCP procedure to which is added teleguidance, is $91.6 \%$. Moreover, we could estimate a $97.9 \%$ probability that teleguidance was cost-saving. The average overall expected savings per patient, in this base case scenario, was 990 SEK (111.2 USD) $(95 \% \mathrm{Cl}$ : 959 to 1,021 SEK). When teleguidance is applied during a routine ERCP the probability of transitions into states associated with lower Utility and additional costs decreased. In a similar situation the QALY increased and the total costs decreased. However, this is not the case under all conditions and for all pa- tients, which is also clarified by the outcomes in the cost-effectiveness plane in $\mathbf{F i g . 3}$, where $0.2 \%$ of the possible combinations of parameters result in outcomes were associated with a lower QALY and increased costs, suggesting that alternative strategies should be followed. The average cost saving for the base case scenario still compensated for the additional costs carried by the equipment and the initial training of the endoscopist.

Currently it was assumed that the novice-trainee should use the teleguidance equipment and have access to the service during a 3-year period. Given a depreciation time of the equipment of 3 years, an initial cannulation success rate of the novicetrainee of $85 \%$ and that of the expert to be $99 \%$, the $t_{\text {traditional }}$ to 10 years, the $t_{\text {TM }}$ to 5 year and $t$ defined as 3 years (see also - Fig.2), these preconditions resulted in cost savings of 780,401 SEK $(87,686$ USD) only due to decrease in number of re-interventions and changes in strategies following cannulation failure. In this case the novice-trainee performed 50 ERCP procedures per year. The model recalculated this estimated cost saving whenever any of the above variables were changed. To estimate the total cost saving after 10 years, one also has to consider the decrease in surgery-reintervention related complications that follow an enhanced cannulation success rates, as reflected by the steeper slope of the learning curve as well as the decrease in the duration of each individual ERCP procedure.

\section{Sensitivity and scenario analyses}

Concerning the incremental cost-effectiveness ratio, a one-way sensitivity analysis revealed that the final parameters always centered around the 20 most sensitive parameters, when changing the respective parameters within their $95 \%$ confidence intervals, one at a time. This is mainly due to the fact that these parameters are contained within the integrated predictive models.

The economic impact of teleguidance during an ERCP procedure, in six hypotethical centers and under nine different conditions, are presented in Tables1-s10 given in the appendix. In these tables the average impact on incremental costs and incremental QALYs are given in addition to the incremental net benefit (INB) based on a willingness to pay threshold of 500,000 SEK $(56,180$ USD)/QALY. The probability of cost-effectiveness, given this threshold together with the probability of cost saving and QALY increase, is also summarized in these tables.

These calculations are based on the widely applied strategy, at a low- to medium-volume hospital, to proceed with redo ERCP in case of cannulation failure whereupon the clinician then switches to PTC and first thereafter refers the patient to an expert. If an endoscopist at such a center was performing an average of 50 ERCPs per year, data suggested that teleguidance emerged as cost-effective when applied to the majority of patients, already when $25 \%$ expert knowledge was transferred and the average procedure complexity was equal to four.

In a case in which a endoscopist was doing 100 ERCPs per year, and followed the same strategy in case of failure, teleguidance became a cost-effective and cost-saving strategy when 
at least $50 \%$ expert knowledge was transferred at an average complexity score of $\geq 2$.

If the endoscopist was performing an average 50 ERCPs per year in a center with a strategy to send patients to PTC and then to an expert in case of failure, teleguidance was a cost-effective and cost saving option when at least $50 \%$ expert knowledge was transferred and the average complexity score was $\geq 2$ (for details see appendix).

Yet another common redo strategy can be followed at a lowto medium-volume hospitals, in case of cannulation failure, i.e. to refer the patient directly to an expert. In the situation in which an endoscopist performed, on an average 50 ERCPs per year, our analyses suggested that teleguidance became cost-effective, and a cost-saving option for the majority of patients, when $25 \%$ expert knowledge was transferred with an the average complexity score of $\geq 2$ (for details see appendix).

When it came to QALY increase, teleguidance became the preferable option for the majority of patients under all conditions displayed in these tables (see supplement-appendix).

\section{Discussion}

The objective of the current study was to address the health economic issues connected with implementation of teleguidance, taking advantage of the latter's inborn potential to facilitate training and quality assurance in routine clinical ERCP practices. A probabilistic health economic model was constructed to investigate for which type of hospital and under which conditions teleguidance could be the preferred option to minimize treatment failure and thereby control the expenditures. The current model was designed to mimic and incorporate the wide clinical spectrum of complexities exposed to when carrying out respective procedures and thereby as closely as possible reflect the daily activities seen in low- and medium-volume hospitals [7]. Primary outcome measures for the evaluation were, as in most corresponding analyses, cost savings and increase inQALY. QALY is a vital parameter by which it is possible to weigh different medical efforts against each other. The relevance of QALY assessment is based on the fact that it is incomplete to solely estimate how many years extra a specific medical efforts can offer, but also take into account the quality of these years. Our model was also constructed to allow for inclusion of the most significant management options in the clinical course and management of these patients [8-34]. For the experimental setting we used a base case scenario hospital, representing a low-volume center where the endoscopist performed 50 ERCPs Annually. In case of transpapillary cannulation failure, the most common strategy in such hospitals is to proceed with a redo ERCP and then to PTC and if the clinical problem remains unsolved, send the patient to an expert. For the same base case, the average complexity grade was set to 1.98 (4 graded scale) and the amount of expert knowledge transferred to $50 \%$. For a willingness-to-pay threshold, which in most corresponding clinical situations amounts to 500,000 SEK (56,180 USD)/ QALY, the probability of cost-effectiveness for the base case scenario was found to be as high as $98.9 \%$. Moreover, the probability that teleguidance is cost-saving was more than $97.9 \%$ and the overall expected saving per patient was 990 SEK (111.2 USD) (albeit with quite wide confidence intervals). In fact our calculations suggested that TM was cost-effective irrespective of the level of the willingness to pay. At the other end of the spectrum we observed that in only $0.2 \%$ of the various scenarios, a reduced QALY and increased costs prevailed. The immediate implications of these results are that TM has to be seriously considered to for integration in future teaching curriculums in advanced upper gastrointestinal endoscopy.

Sensitivity analyses are of vital importance for clinical and methodological relevance of studies like these. The economic impact of teleguidance during an ERCP was currently assessed for six hypotethical hospitals under nine different clinical situations. We reported in detail, based in these different case scenarios, the average impact on incremental costs and incremental QALYs in addition to the incremental net benefit (INB) everything based on a willingness to pay threshold of 500,000 SEK $(56,180$ USD)/QALY. When it came to QALY increase, teleguidance was the preferable option for the majority of patients. Concerning the incremental cost-effectiveness ratio, a oneway sensitivity analysis revealed that the final parameters always centered around the 20 most sensitive parameters, when changing the respective parameters from their 2.5 percentile to their 97.5 percentile one at a time. As expected the predominant factors determining the outcomes were the cost of the teleguidance, complexity of the procedure, duration of the procedure, and length of hospital stay.

There are obvious limitations, uncertainties, and potential weaknesses connected with the current analyses, such as the cut-off levels for the Number of cases-procedures done on an annual basis in low- to medium-volume hospitals, the case mix and the complexity score of the actual patients. In addition to that, it can be discussed how training is structured and implemented at the studied hospital as well as the time frames that should be applied to most accurately mimic common clinical practice. It is generally recognized that cannulation success rates are reliable proxies for the level of training and expertise when carrying out ERCP [36]. Robust figures on these topics emerge from national registers that cover the entire population of a country [37]. In addition, among endoscopists, it is well established what is meant by a "difficult cannulation" situation [36]. Second, the complexity scorings have to be based on a comprehensive review of the available literature. Corresponding figures can also be obtained from national registers, as indicated above, albeit that such details from each individual patient cannot be entered into these databases for practical and other logistical reasons. No doubt the calculated figures on complication rates, following the different strategies in the models, are based on a huge amount of data captured from the literature, which now have to be cross-checked with prospectively collected information from the Swedish national register, covering more than $95 \%$ of all ERCPs done per year [37]. These figures are of crucial importance because complications are central for cost assessment of procedures, in which situations also consultations and guidance over the telemedicine medium obviously carry a significant potential to be cost-saving. Another factor contained in the different scenarios was 
the amount of knowledge and experience transmitted during a teleguidance session. It is inevitable that such estimates are partly subjective and eminence- rather than evidence-based. We made efforts to be as conservative and cautious as possible to minimize risk of overestimating the clinical and economical consequences. However, an ongoing prospective clinical trial will cast further light also on this issue.

The current models, trying to explore the health economic rationale behind teleguidance support for ERCP procedures in low-volume peripheral hospitals, may have their counterpart in dissemination of surgical competence to remote hospitals, e. g. during implementation of minimally invasive surgical procedures [38]. Early experiences have been reported on the potential role of telepresence in delivering and maintaining highquality surgical care in remote hospitals even in a period of rapid advancements in surgical techniques and technologies. There is no doubt that with evolution of telecommunication technology, and corresponding teleguidance practice, effective means can be delivered to foster advanced surgical care in community hospitals [39]. These considerations are highly relevant also for the advanced technologies within the field of gastrointestinal endoscopy $[40,41]$. Teleguidance assistance allows for "on-the-job" training without the drawbacks associated with lengthy travel. Under similar circumstances we were unable to detect a trend toward shorter procedure times with expanding experience. On the other hand, the shift in case selection during the learning period suggested more technically challenging cases during the latter part of the study period.

One dimension that also has to be taken into consideration, although not amenable to be defined in economic terms, is the experiences gained from the patients (plus their relatives) who have been educated and informed concerning the potential risks and benefits of teleguidance. It seems, however, that these individuals are highly receptive to being part of implementation of corresponding novel technologies [38-41]. A similar therapeutic strategy allowed them to benefit from transmission of expertise, while remaining in their local hospital under the care of their local surgeon or endoscopist.

\section{Conclusion}

In conclusion, teleguidance during ERCP seems to be the preferable option in many low- to medium-volume hospitals, as defined by a variety of different clinical situations. The main mechanisms behind the effects of teleguidance was a positive impact on major clinical outcome variables, leading to an increase in QALY and a decrease in costs.

\section{Competing interests}

The authors declare that they have no conflict of interest.

\section{References}

[1] Boanca C, Rafiq A, Tamariz F et al. Remote video management for intraoperative consultation and surgical telepresence. Telemed J E Health 2007; 13: 603-607

[2] Haidegger T, Szandor ], Benyo Z. Surgery in space: the future of robotic telesurgery. Surg Endosc 2011; 25: 681-690

[3] Lee SP, Lee HL, Hahm JS et al. International live endoscopic multichannel demonstration using superfast broadband internet connections. Clin Endosc 2012; 45: 73-77

[4] Shimizu S, Itaba S, Yada S et al. Significance of telemedicine for video image transmission of endoscopic retrograde cholangiopancreatography and endoscopic ultrasonography procedures. J Hepatobiliary Pancreat Sci 2011; 18: 366-374

[5] Barkun AN, Adam V, Martel M et al. Partially covered self-expandable metal stents versus polyethylene stents for malignant biliary obstruction: A cost-effectiveness analysis. Can J Gastroenterol Hepatol 2015; 29: 377-383

[6] McKaigney J, Sandha GS. The clinical impact and cost implications of endoscopic ultrasound on the use of endoscopic retrograde cholangiopancreatography in a Canadian university hospital. Can J Gastroenterol 2008; 22: 138-142

[7] Pahlsson HI, Groth K, Permert J et al. Telemedicine: an important aid to perform high-quality endoscopic retrograde cholangiopancreatography in low-volume centers. Endoscopy 2013; 45: 357-361

[8] Andriulli A, Loperfido S, Napolitano G et al. Incidence rates of postERCP complications: a systematic survey of prospective studies. Am J Gastroenterol 2007; 102: 1781-1788

[9] Barthet N, Lesavre A, Desjeux M et al. Complications of endoscopic sphincterotomy: results from a single tertiary referral center. Endoscopy 2002; 34: 991-997

[10] Boender ], Nix GA, de Ridder MA et al. Endoscopic papillotomy for common bile duct stones: factors influencing the complication rate. Endoscopy 1994; 26: 209-216

[11] Chen YK, Foliente RL, Santoro M] et al. Endoscopic sphincterotomyinduced pancreatitis: increased risk associated with nondilated bile ducts and sphincter of Oddi dysfunction. Am J Gastroenterol 1994; 89: 327-333

[12] Choudari CP, Sherman S, Fogel EL et al. Success of ERCP at a referral center after a previously unsuccessful attempt. Gastrointest Endosc 2000; 52: 478-483

[13] Christensen M, Matzen P, Schulze S et al. Complications of ERCP: a prospective study. Gastrointest Endosc 2004; 60: 721-731

[14] Christoforidis E, Goulimaris I, Kanellos I et al. Post-ERCP pancreatitis and hyperamylasemia: patient-related and operative risk factors. Endoscopy 2002; 34: 286-292

[15] Deans GT, Sedman P, Martin DF et al. Are complications of endoscopic sphincterotomy age related? Gut 1997; 41: 545-548

[16] Dickinson RJ, Davies S. Post-ERCP pancreatitis and hyperamylasaemia: the role of operative and patient factors. Eur J Gastroenterol Hepatol 1998; 10: 423-428

[17] Freeman ML, Nelson DB, Sherman S et al. Complications of endoscopic biliary sphincterotomy. N Engl J Med 1996; 335: 909-918

[18] Köklü S, Parlak E, Yüksel O et al. Endoscopic retrograde cholangiopancreatography in the elderly: A prospective and comparative study. Age Ageing 2005; 34: 572-577

[19] Leese T, Neoptolemos JP, Carr-Locke DL. Successes, failures, early complications and their management following endoscopic sphincterotomy: results in 394 consecutive patients from a single centre. $\mathrm{Br}$ J Surg 1985; 72: 215-219 
[20] Harewood GC, Baron TH, LeRoy A] et al. Cost-effectiveness analysis of alternative strategies for palliation of distal biliary obstruction after a failed cannulation attempt. Am J Gastroenterol 2002; 97: 1701-1707

[21] Brown LM, Rogers SJ, Cello J et al. Cost-effective treatment of patients with symptomatic cholelithiasis and possible common bile duct stones. J Am Coll Surg 2011; 212: 1049-1060

[22] Alhayaf N, Lalor E, Bain V et al. The clinical impact and cost implication of endoscopic ultrasound on use of endoscopic retrograde cholangiopancreatography in a Canadian university hospital. Can J Gastroenterol 2008; 22: 138-142

[23] Vergel YB, Chilcott J, Kaltenthaler E et al. Economic evaluation of MR cholangiopancreatography compared to diagnostic ERCP for the investigation of biliary tree obstruction. Int J Surg 2006; 4: 12-19

[24] Sullivan PW, Slejko JF, Sculpher M] et al. Catalogue of EQ-5D scores for the United Kingdom. Med Decis Making 2011; 31: 800-804

[25] Vandervoort J, Soetikno RM, Tham TC et al. Risk factors for complications after performance of ERCP. Gastrointest Endosc 2002; 56: 652656

[26] Tzovaras G, Shukla P, Kow L et al. What are the risks of diagnostic and therapeutic endoscopic retrograde cholangiopancreatography? Aust N Z J Surg 2000; 70: 778-782

[27] Tanner AR. ERCP: present practice in a single region. Suggested standards for monitoring performance. Eur J Gastroenterol Hepatol 1996; 8: 145-148

[28] Suissa A, Yassin K, Lavy A et al. Outcome and early complications of ERCP: a prospective single center study. Hepatogastroenterology 2005; 52: 352-355

[29] Sherman S, Ruffolo TA, Hawes RH et al. Complications of endoscopic sphincterotomy. A prospective series with emphasis on the increased risk associated with sphincter of Oddi dysfunction and nondilated bile ducts. Gastroenterology 1991; 101: 1068-1075

[30] Rabenstein T, Schneider HT, Bulling D et al. Analysis of the risk factors associated with endoscopic sphincterotomy techniques: preliminary results of a prospective study, with emphasis on the reduced risk of acute pancreatitis with low-dose anticoagulation treatment. Endoscopy 2000; 32: 10-19
[31] Ong TZ, Khor JL, Selamat DS et al. Complications of endoscopic retrograde cholangiography in the post-MRCP era: a tertiary center experience. World J Gastroenterol 2005; 11: 5209-5212

[32] Masci E, Toti G, Mariani A et al. Complications of diagnostic and therapeutic ERCP: a prospective multicenter study. Am J Gastroenterol 2001; 96: 417-423

[33] Loperfido S, Angelini G, Benedetti G et al. Major early complications from diagnostic and therapeutic ERCP: a prospective multicenter study. Gastrointest Endosc 1998; 48: 1-10

[34] Lal D, Lane M, Wong P. Complications of endoscopic retrograde cholangiopancreatography. N Z Med J 2003; 116: U496

[35] Statistics Sweden. [Online] Available: http://www.scb.se/en_/finding-statistics/statistics-by-subject-area/population/population-composition/population-statistics/\#c_undefined

[36] Halttunen J, Meisner S, Aabakken L et al. Difficult cannulation as defined by a prospective study of the Scandinavian Association for Digestive Endoscopy (SADE) in 907 ERCPs. Scand J Gastroenterol 2014; 49: $752-758$

[37] Enochsson L, Thulin A, Osterberg J et al. The Swedish Registry of Gallstone Surgery and Endoscopic Retrograde Cholangiopancreatography (GallRiks): A nationwide registry for quality assurance of gallstone surgery. JAMA Surg 2013; 148: 471-478

[38] Anvari M. Remote telepresence surgery: the Canadian experience. Surg Endosc 2007; 21: 537-541

[39] Boanca A, Rafiq F, Tamariz V et al. Remote video management for intraoperative consultation and surgical telepresence. Telemed J E Health 2007; 13: 603-607

[40] Barkun AN, Adam V, Martel M et al. Partially covered self-expandable metal stents versus polyethylene stents for malignant biliary obstruction: A cost-effectiveness analysis. Can J Gastroenterol Hepatol 2015; 29: 377-383

[41] Bhatt A, Abe S, Umaravel A et al. Video-based supervision for training of endoscopic submucosal dissection. Endoscopy 2016; 48: 711-716 